\title{
A rare case of acute hepatitis caused by Komboucha tea
}

\author{
C. Steger, T. Vandamme, P.H. Steger \\ Sint-Vincentiusziekenhuis, Antwerpen, Belgium.
}

\section{To the Editor,}

HILI or "herb-induced" liver injury is a rare cause of acute liver injury related to traditional Asian medicine and herb-based products. Similar to previously published case report by Vanstraelen et al. (1) we present a case of a 55-year-old man with complaints of fatigue since two months, and complaints of nausea, frequent vomiting and a darkening of his urine since one week. He reported taking no other medication than occasionally cetirizine $10 \mathrm{mg}$. In the last 6 months he denied taking any other medication. Prior history of hay fever was known. Patient reported drinking one or two glasses of wine since several years and recently started drinking several glasses of Komboucha tea daily.

At presentation his parameters were stable and on sight mild jaundice was noticed. Further clinical investigation showed no other abnormalities with normal abdominal investigation.
Drug-induced hepatotoxicity could be excluded since the patient's only medication intake was cetirizine, which causes hepatic toxicity only in specific, rare cases (2).

Patient was advised to stop drinking Komboucha tea and alcohol. Re-evaluation after 1 and 6 months showed disappearance of the complaints with normalisation of most of the blood results after six months (Table 1).

The above-mentioned patient was diagnosed with acute toxic hepatitis caused by Komboucha tea. Similar to the case report by Vanstraelen et al. (1) there was a chronic association between the drinking of Komboucha tea and the start of the symptoms. Given the mild clinical presentation and quick recuperation after cessation, no biopsy was made and so the diagnosis remains uncertain. The disappearance of the symptoms after cessation of drinking Komboucha tea favours the above diagnosis, but other additive factors such as alcohol can't be fully excluded.

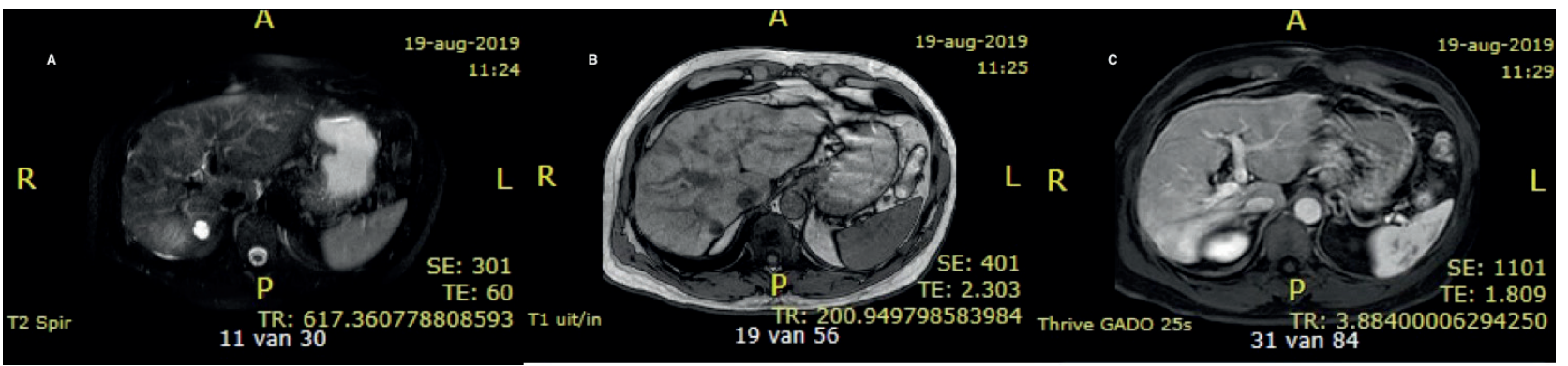

Figure 1.

Laboratory findings showed the presence of mixed elevated values of liver enzymes with normal value of lipase (Table 1). Further investigation consisting of abdominal ultrasound and MRI scan with MRCPsequence showed an inhomogeneous right liver lobe, but no signs of steatosis or cirrhosis, no liver or pancreatic masses and no sign of cholestatic injury or obstruction (Figure 1).

Biochemical testing to eliminate viral and autoimmune hepatitis was conducted and showed no abnormalities. Malignancy was excluded by the absence of abnormalities on diagnostic imaging and the normal values of cancer biomarkers. Further diagnostic work up revealed no sings of underlying metabolic disease such as Wilson's disease or hemochromatosis (Table 2). Screening for metabolic syndrome was negative.
Following the gaining popularity of traditional Asian lifestyle and the more frequent consumption of products like camellia sinensis, which is the main and most likely hepatotoxic, ingredient in Komboucha tea, more knowledge and vigilance about HILI is needed in our day to day practice, certainly when there is a combined use with known hepatotoxic products such as alcohol (3).

\section{Conflict of interest}

None to declare.

\footnotetext{
Correspondence to: Charlotte Steger, Van Putlei 64, bus 8, 2000 Anterpen, Belgium. Phone : +32 0492085588. Fax : +32 (0)32852885.

E-mail : charlottesteger@hotmail.com

Submission date : 20/08/2020

Acceptance date : $24 / 09 / 2020$
} 


\section{References}

1. VANSTRAELEN, S., RAHIER, J., GEUBEL, A.P., 2008. Jaundice as a misadventure of a green tea (camellia sinensis) lover : a case report. Acta Gastroenterol. Belg., 71 : 409-412.
2. LIVERTOX: CLINICAL AND RESEARCH INFORMATION ON DRUGINDUCED LIVER INJURY. Bethesda (MD) : National Institute of Diabetes and Digestive and Kidney Diseases; 2012-. Cetirizine. Updated 2017 Jan 16. https://www.ncbi.nlm.nih.gov/books/NBK548420/

3. STICKEL, F., KESSEBOHM, K., WEIMANN, R., SEITZ, H.K., 2011. Review of liver injury associated with dietary supplements. Liver Int., 31 : 595-605.

Table 1. - Laboratory results

\begin{tabular}{|c|c|c|c|c|}
\hline & 19/08/2019 & $04 / 09 / 2019$ & $27 / 01 / 2020$ & Reference value \\
\hline hematocrit & 45,3 & 43,5 & 42,8 & $39-51 \%$ \\
\hline MCV & 87,6 & 90,6 & 93,9 & $79,1-99,0 \mathrm{fl}$ \\
\hline МCH & 31,1 & 31,0 & 31,8 & $26,7-34,5 \mathrm{pg}$ \\
\hline МCHC & 35,5 & 34,3 & 33,9 & $33,2-36,9 \mathrm{~g} / \mathrm{dl}$ \\
\hline leucocytes & 4,5 & 4,5 & 6,98 & $3,30-9,30 \times 1000 / \mathrm{mm}^{\wedge} 3$ \\
\hline lymphocytes & 22,1 & 38,4 & 38,0 & $17,6-47,6 \%$ \\
\hline monocytes & 14,3 & 12,6 & 9,7 & $4,0-11,3 \%$ \\
\hline thrombocytes & 94 & 74 & 123 & $131-360 \times 1000 / \mathrm{mm}^{\wedge} 3$ \\
\hline APTT & - & 36,4 & - & $24,8-34,4 \mathrm{sec}$ \\
\hline PT & - & 82 & - & $70-150 \%$ \\
\hline INR & - & 1,25 & - & $0,80-1,20$ \\
\hline urea & 26 & 22 & 20 & $18-55 \mathrm{mg} / \mathrm{dl}$ \\
\hline creatinine & 0,90 & 0,90 & 0,81 & $0,73-1,18 \mathrm{mg} / \mathrm{dl}$ \\
\hline eGFR & $>60$ & $>60$ & $>60$ & $>60 \mathrm{ml} / \mathrm{min} / 1,73 \mathrm{~mm} 2$ \\
\hline uric acid & 4,7 & 4,2 & 5,1 & $3,5-6,0 \mathrm{mg} / \mathrm{dl}$ \\
\hline sodium & 140 & - & - & $136-145 \mathrm{mmol} / \mathrm{L}$ \\
\hline potassium & 4,0 & - & - & $3,5-5,1 \mathrm{mmol} / \mathrm{L}$ \\
\hline chloride & 107 & - & - & $98-107 \mathrm{mmol} / \mathrm{L}$ \\
\hline bicarbonate & 25 & - & - & $22-29 \mathrm{mmol} / \mathrm{L}$ \\
\hline calcium & 2,36 & - & - & $2,10-2,52 \mathrm{mmol} / \mathrm{L}$ \\
\hline phosphate & 0,78 & - & - & $0,71-1,52 \mathrm{mmol} / \mathrm{L}$ \\
\hline magnesium & 0,84 & - & - & $0,66-1,07 \mathrm{mmol} / \mathrm{L}$ \\
\hline iron & 240 & 218 & 134 & $65-175 \mathrm{mg} / \mathrm{dl}$ \\
\hline transferrin & 2,01 & 1,78 & 2,29 & $1,74-3,64 \mathrm{~g} / \mathrm{L}$ \\
\hline transferrin saturation & 85 & 87 & 41 & $16-45 \%$ \\
\hline ferritin & 10310 & 3363 & 279 & $22-275 \mu \mathrm{g} / \mathrm{L}$ \\
\hline cholesterol & 263 & - & 204 & $\mathrm{mg} / \mathrm{dl}$ \\
\hline triglycerides & 190 & - & - & $\mathrm{mg} / \mathrm{dl}$ \\
\hline bilirubin total & 3,9 & 2,8 & 0,6 & $0,3-1,2 \mathrm{mg} / \mathrm{dl}$ \\
\hline bilirubin direct & 2,2 & 1,5 & 0,3 & $0,0-0,5 \mathrm{mg} / \mathrm{dl}$ \\
\hline bilirubin indirect & 1,7 & 1,3 & 0,3 & $<0,7 \mathrm{mg} / \mathrm{dl}$ \\
\hline lactate & 2,14 & - & - & $0,5-2,20 \mathrm{mmol} / \mathrm{L}$ \\
\hline AST & 604 & 247 & 33 & $<34 \mathrm{U} / \mathrm{L}$ \\
\hline ALT & 1070 & 394 & 44 & $<55 \mathrm{U} / \mathrm{L}$ \\
\hline Y-gt & 843 & 681 & 147 & $11-59 \mathrm{U} / \mathrm{L}$ \\
\hline ALP & 171 & 146 & 82 & 43-160 IU/L \\
\hline LDH & - & 354 & - & $125-220 \mathrm{U} / \mathrm{L}$ \\
\hline lipase & 48 & 34 & - & $<78 \mathrm{U} / \mathrm{L}$ \\
\hline CRP & 3 & - & - & $<5 \mathrm{mg} / \mathrm{dl}$ \\
\hline
\end{tabular}

$\mathrm{MCV}$ : mean corpuscular volume, $\mathrm{MCH}$ : mean corpuscular hemoglobin, $\mathrm{MCHC}$ : mean corpuscular hemoglobin concentration, APTT : activated partial thromboplastin time, PT : prothrombin time, INR : international normalized ratio, eGFR : estimated glomerular filtration rate, AST : aspartate aminotransferase, ALT : alanine aminotransferase, Y-gt : gamma-glutamyl transpeptidase, ALP : Alkaline phosphatase, LDH : Lactate dehydrogenase, CRP : C-reactive protein. 
Table 2. - Results etiological investigation

\begin{tabular}{|l|c|c|}
\hline & Result & Reference value \\
\hline IGA total & 6,03 & $0,63-4,84 \mathrm{~g} / \mathrm{L}$ \\
\hline IGG total & 11,09 & $5,40-18,22 \mathrm{~g} / \mathrm{L}$ \\
\hline IGM total & 1,97 & $0,22-2,40 \mathrm{~g} / \mathrm{L}$ \\
\hline ANF & negative & - \\
\hline AMA & negative & - \\
\hline Anti-LKM antibody & negative & - \\
\hline Anti-SMC antibody & negative & - \\
\hline Toxoplasmosis IGG & negative & - \\
\hline Toxoplasmosis IGM & negative & - \\
\hline HIV & negative & - \\
\hline Hepatitis A IGM & negative & - \\
\hline Hepatitis B surface antibodies & negative & - \\
\hline Hepatitis C antibodies & negative & - \\
\hline Hepatitis E IGM screening & negative & - \\
\hline Mononucleosis screening & negative & - \\
\hline EBV IGG & positive $(15,45 \mathrm{index})$ & - \\
\hline EBV IGM & negative & - \\
\hline CMV IGG & positive $(64,4 \mathrm{U} / \mathrm{ml})$ & $0,0-5,0 \mu \mathrm{g} / \mathrm{L}$ \\
\hline CMV IGM & negative & $0,0-37,0 \mathrm{KU} / \mathrm{L}$ \\
\hline CEA & 3,8 & $<8,87 \mu \mathrm{g} / \mathrm{L}$ \\
\hline CA 19.9 & 13,9 & $80-140 \mathrm{mg} / \mathrm{dl}$ \\
\hline AFP & 35,73 & $0,22-0,58 \mathrm{~g} / \mathrm{L}$ \\
\hline copper & 74 & \\
\hline ceruloplasmin & 0,23 & - \\
\hline
\end{tabular}

IGA : immunoglobulin A, IGG : immunoglobulin B, IGM : immunoglobulin M, ANF : antinuclear factor, AMA : anti-mitochondrial antibodies, Anti-LKM antibody : anti-liver-kidney microsomal antibody, Anti-SMC antibody : Anti-smooth muscle antibodies, PCR : Polymerase chain reaction, EBV : Epstein-Barr-virus, CMV : Cytomegalovirus, CEA : carcinoembryonic antigen, CA 19.9 : cancer antigen 19-9, AFP : alpha-fetoprotein. 